Instituto Internacional de Investigación y Desarrollo Tecnológico Educativo INDTEC, C.A.

DOI: https://doi.org/10.29394/Scientific.issn.2542-2987.2019.4.13.4.78-89

OAI-PMH: http://www.indteca.com/ojs/index.php/Revista Scientific/oai

Artículo Original / Original Article

\title{
Cultivo de Cacao (Theobroma cacao linnaeus) como Rubro para la Sustentabilidad de los Suelos
} (Investigación en Desarrollo)

Autor: Franklin Rene Guerrero Guerrero Universidad Pedagógica Experimental Libertador, UPEL franklinguerrero77@gmail.com Barinas, Venezuela https://orcid.org/0000-0002-9971-5270

\section{Resumen}

La presente investigación, establece como objetivo general proponer el Cultivo de cacao (Theobroma cacao linnaeus) como rubro para la sustentabilidad de los suelos a los Agricultores del Caserío El Tesoro, parroquia Ciudad Bolivia, municipio Pedraza, estado Barinas, fija su enfoque investigativo en el paradigma cuantitativo, como un proyecto especial, basado en el diseño de Campo de carácter exploratorio a ser ejecutado en cuatro etapas: 1. Diagnóstico, 2. Diseño, 3. Aplicación, y 4. Sistematización. Se aplicará la observación directa en prácticas de campo, además de encuestas de tipo cuestionario con una escala de frecuencia tipo Likert de cinco (5) alternativas de respuestas a los productores del caserío antes señalado; la validez se estará ejecutando con el juicio de expertos, la confiabilidad se medirá por el Alpha de Cronbach para determinar el grado de legitimidad y efectividad de los instrumentos. El análisis de los datos recolectados se hará por medio de tablas y gráficos con su respectiva descripción para su mejor interpretación. Al ser un estudio en proceso, se espera que los resultados a obtener transformen positivamente la realidad de la comunidad ya que los productores deterioran el suelo con sus prácticas agrícolas y es necesario realizar cambios que permitan tener una cultura ambientalista que genere beneficios tanto económicos como ecológicos en pro de la sustentabilidad.

Palabras clave: cultivo; agricultura de subsistencia; tierra agrícola.

Fecha de Recepción: 28-12-2018
Fecha de Aceptación: 26-04-2019
Fecha de Publicación: 05-08-2019 


\section{Cultivation of Cocoa (Theobroma cacao linnaeus) as an Item for the Sustainability of Soils \\ (Project in Development)}

Date Received: 28-12-2018
Date Acceptance: 26-04-2019
Date Publication: 05-08-2019 


\section{Introducción}

La sustentabilidad de los suelos se ha tornado en un tema de mucha importancia en ámbito agrícola, ya que se está evidenciando la degradación de los mismos en los últimos años, a través de los bajos rendimientos de producción en zonas que anteriormente tenían excelentes características de fertilidad. Todo ello, ha llevado a la necesidad de adoptar técnicas agrícolas amigables con el ambiente, considerando que la agricultura convencional ha ocasionado gran parte de los problemas en su afán de lograr mayor producción por unidad de superficie de suelo para cubrir la demanda de alimentos que en los últimos años se ha elevado cuantitativamente.

El uso continuo e inconsciente de agroquímicos y maquinaria en los campos ha incrementado los problemas de erosión, compactación y aumento de salinidad trayendo como consecuencia efectos negativos al ambiente, sobre todo a la diversidad biológica de estos ecosistemas, lo que trae como principal problema, una degradación en sus características químico-físicas. Es importante hacer énfasis en la necesidad de buscar opciones que contribuyan a la conservación del ambiente y la sustentabilidad de los suelos utilizando cualquier método agroecológico, una de estas opciones puede ser el establecimiento de cultivos perennes, tal es el caso del cacao (Theobroma cacao linnaeus) que además de ser un cultivo agroforestal de fácil manejo, su producción (grano de cacao) tiene gran demanda nacional e internacional lo que se traduce en beneficios económicos a las familias del sector rural.

Además de lo antes mencionado proporciona materia orgánica por medio de la cobertura vegetal, promoviendo un crecimiento y equilibrio en los organismos formadores de suelo, suprimiendo en lo posible el uso de maquinaria y agroquímicos, evitando así los problemas de erosión, compactación entre otros. Es oportuno mencionar, que el presente estudio que se establece como objetivo general proponer el cultivo de cacao (Theobroma cacao linnaeus) como rubro para la sustentabilidad de los suelos a los 
Agricultores del Caserío El Tesoro, parroquia Ciudad Bolivia, municipio Pedraza, estado Barinas, está inscrito en la línea de investigación del Instituto de Mejoramiento Profesional del Magisterio de la Universidad Pedagógica Experimental Libertador Barinas "Educación, Desarrollo y Gestión Comunitaria", donde se propone el Cultivo de cacao como rubro para la sustentabilidad de los suelos dirigido a los Agricultores del caserío El Tesoro, parroquia Ciudad Bolivia, municipio Pedraza, estado Barinas.

\section{Fundamentación Teórica}

\subsection{Cultivo de Cacao}

La producción de cacao a nivel mundial, está estimada alrededor de 3.600.000 toneladas de grano, dicho en la Organización Internacional del Cacao (ICCO, 2006): El aporte que provee el cultivo de cacao gracias a sus productores de menor y media escala, ha venido evolucionando positivamente en no solo en el sector agrario, sino en el económico, pues cerca de 3 millones de toneladas son cosechadas cada año por los mismos (pág. 6); así pues, en la actualidad, 50 países tropicales de los continentes de América, África y Asia se han convertido en productores de dicho rubro, contribuyendo con la transformación de tierras y bosques con su labor agrícola, lo que equivale al $85 \%$ de la producción mundial anualmente.

Según Rice (2000), citado por Cabezas, Mavisoy, Ballesteros y Somarriba (2009): A nivel social, los pequeños productores aprovechan la explotación con relación a la venta y el consumo de especies frutales y maderables, estas actividades, proveen empleos y por ende, recursos suplementarios de ingresos activos permitiendo que se estabilicen a nivel económico ante las fluctuaciones en los costos del comercio del cacao (pág. 10).

Al respeto, Dubois (2007), expresa que: el cacao, es producido en medio de sistemas agroforestales ricos en variedades de especies, pues 
donde se cultiva, también hay presencia de otros rubros que mejoran la calidad de su fruto de forma natural, toda esta actividad agrícola, favorece a los pueblos en cuanto a economía y cultura (pág. 8); sin contar con la biodiversidad de vegetales y animales (mamíferos, coleópteros) que se producen. Para Harvey, González y Somarriba (2006): estas especies presentes en los cultivos de cacao, sirven como agentes polinizadores sin necesidad del uso de agroquímicos, ofreciendo entonces en el mercado comercial, un producto orgánico natural y de calidad (pág. 555).

Según los autores antes señalados, el cultivo de cacao ha sido y sigue siendo un rubro de gran relevancia en el desarrollo del sector rural, haciendo hincapié en el papel que representan los pequeños productores en el aporte a la producción mundial tomando en cuenta que gran parte de esa producción procede de este conjunto. Es un cultivo de gran importancia ya que se desarrolla en sistema agroforestales logrando establecer una diversidad biológica dentro de los cultivares a través de la creación de bosques.

\subsection{Variedades de cacao}

De acuerdo Brickell, et al. (2009), en el Código Internacional de Nomenclatura de las plantas cultivadas, expone que: el termino variedad es el mismo que cultivar, cuando aplicado a un conjunto de individuo que muestran diferencias genéticas, pero que tienen una o más características por las cuales pueden ser diferenciados de otros cultivares o variedades (pág. 6).

Bajo esta definición el cacao (Theobroma cacao linnaeus) en Venezuela tiene varias presentaciones; EI criollo: caracterizado por presentar cotiledones blancos o con una ligera coloración violeta pálida a rosado y de mucílago dulce, superficie del fruto muy rugosa con diez surcos, punta atenuada y corteza fácil de cortar. El amazónico o forastero: de almendras aplanados cotiledones de color púrpura, su superficie generalmente lisa y pericarpio grueso y resistente al corte. EI Trinitario: generado por cruces 
naturales o hibridaciones entre los dos grupos anteriores. Actualmente se considera a las estribaciones de la cordillera andina y a la cuenca del lago de Maracaibo como la cuna del cacao criollo venezolano desde donde fue distribuido de las congregaciones religiosas.

Es importante tomar en cuenta las variedades que se van a utilizar en el establecimiento de una plantación de cacao, ya que cada una de ellas tienen distintas exigencias de adaptación según la región y manejo en sus labores culturales, en la mayoría de los casos la variedades más difundidas en Venezuela son las criollas, que se caracterizan por producir una almendra con las mejores características para producción de chocolates de excelente calidad, con la desventajas que estas plantas son muy susceptibles a las principales enfermedades que atacan a este cultivo.

\subsection{Técnicas de manejo sustentable del suelo}

El severo impacto que tienen en el ambiente las técnicas convencionales de manejo de suelos ha incentivado al desarrollo de técnicas alternativas de corte agroecológico las cuales no solo son respetuosas del ambiente, sino además inducen la mejora y preservación de la fertilidad y productividad de los suelos. Según Calva, et al. (2007), éstas técnicas incluyen: sistema de control biológico para evitar el uso d plaguicidas; abonos verdes y biofertilizantes a base de bacterias y hongos que reducen el uso de fertilizantes químicos; acolchados y cultivos de cobertura para evitar la erosión; sistema de labranza de conservación que evitan la compactación de los suelos, y policultivos que aprovechen de manera más eficiente los recursos edáficos y al mismo tiempo reduzcan el riesgo de plagas y enfermedades (pág. 14). Estos productos agrícolas libre de insumos artificiales están generando un mercado emergente, el de la agricultura "orgánica", que resulta cada vez más competitivo.

Es importante recalcar que todas las actividades agrícolas donde se 
sustituyan de manera considerable el uso de agroquímicos y maquinaria por técnicas y productos agroecológicos, no solo contribuyen a mantener la fertilidad conservación de los suelos y el ambiente en general, sino que también ayuda al bienestar de la población, al producir alimentos sanos libres de agro-tóxicos.

\section{Materiales y métodos}

El presente estudio, sienta su base metodológica en el tipo de investigación en la Modalidad de Proyecto Especial que según el Manual de Trabajos de Grado de Especialización y Maestría y Tesis Doctorales de la Universidad Pedagógica Experimental Libertador (UPEL, 2006a): "son trabajos que lleven a creaciones tangibles, susceptibles de ser utilizadas como soluciones a problemas demostrados, respondan a necesidades e intereses de tipo cultural" (pág. 23).

Por lo antes mencionado, se empleará una indagación, planificación, intervención y evaluación para transformar la realidad observada que en este caso específico sería el deterioro de los suelos del caserío por la utilización de maquinarias y químicos para la agricultura, además de otras prácticas que maltratan los suelos fértiles de la población y causan erosiones casi irreparables en esta capa tan importante para cultivar la vida en el planeta. En este sentido, con relación a la aplicación de una investigación bajo la modalidad de proyecto especial, el Manual de la Universidad Pedagógica Experimental Libertador (UPEL, 2006b), expresa que: “...Por su carácter de innovador puede producir un aporte significativo al conocimiento sobre el tema seleccionado y a la cultura..." (pág. 22).

Con apoyo en las citas anteriores, al tratarse de un proyecto especial, se anuncia una producción que dé como resultado de su estudio un producto tangible, que refleje una transformación onto-axiológica del fenómeno en estudio que en este caso es suprimir el deterioro del suelo con la 
implementación de una siembra, en el Manual de la Universidad Pedagógica Experimental Libertador (UPEL, 2006c), se especifica cuatro fases para el proyecto especial: La fase 1. Demostración de la necesidad de la creación o de la importancia del aporte, la cual se trata del diagnóstico que debe demostrar que hay una imperiosa necesidad de realizar la transformación bien sea cultural o de conocimiento (pág. 22); en esta fase, se detectó por medio de la observación, plática con agricultores del caserío y la práctica diaria del investigador la problemática a abordar para hacer una transformación. La fase 2. se trata de la Fundamentación teórica, que sería la ampliación y profundización de las variables del estudio, además del respaldo en diversos autores que hayan realizado investigaciones similares a las temáticas establecidas como objeto de estudio, al final de esta fase se señalan las bases legales y cuadro de operacionalización de variables (pág. 22); para completar esta fase, fue necesario abordar teorías que fundamentan la práctica de cultivos de cacao y la sustentabilidad de los suelos, además se ubicaron y presentaron aportes significativos que otros autores han encontrado como producto de su estudio, de igual forma, se puntualizaron los artículos de las leyes venezolanas que dichas temáticas. En la fase 3. Descripción de la metodología, es una de las más importantes pues es la que le da la tilde de cientificidad, puesto que un estudio sin rigurosidad sistemática no resultaría científicamente confirmable en vista de que carecería de un método de indagación para investigar (pág. 22); en esta fase se declaró la naturaleza, el tipo y diseño de la investigación, las etapas, población y muestra a estudiar, técnicas de recolección y análisis de los datos y por supuesto la validez y confiabilidad de la misma. Finalmente, en la fase 4. Se presenta el Resultado concreto, una vez que se establezcan y cumplan las etapas anteriores, es necesario realizar la intervención y sistematizar detalladamente cada proceso para cerrar el ciclo investigativo presentando el resultado de las acciones implementadas en función de mejorar tanto el método aplicado como la 
situación observada (pág. 22); cuando se culmine esta fase, se publicarán los resultados para que sirva como talante para futuras investigaciones, además, se habrá alcanzado el objetivo de que los agricultores aprendan a aplicar prácticas agrícolas que preserven los suelos evitando su deterioro.

\section{Análisis e Interpretación de los resultados}

Una parte importante de cada proceso que se proponga ser estudiado desde un perspectiva científica en el paradigma cuantitativo es la forma como se analizan los datos recolectados y de qué manera se interpretan pues el objetivo es entender una situación para dependiendo del tipo de investigación hacer una intervención en pro de transformar el fenómeno o la forma de verlo, justificando lo dicho, Hernández, Fernández y Batista (2012), expresan que: "el paradigma cuantitativo establece la recolección y estadística para establecer patrones y comportamiento de una población" (pág. 97).

Para el análisis e interpretación de los datos de esta investigación, serán tabulados y analizados descriptivamente para su entendimiento en función de consolidar los objetivos formulados para promover el Cultivo de cacao como método de conservación de los suelos en el caserío El Tesoro, parroquia Ciudad Bolivia, municipio Pedraza, es preciso decir, que se aplicarán encuestas validadas por medio del Juicio de expertos (2) dos en el área de ambiente y (1) uno de metodología, a los cuales se les hará entrega de una comunicación con un instrumento para que lo validen, tomando en cuenta la redacción, pertinencia, claridad y congruencia de cada ítems, posteriormente se harán ajustes sugeridos por los expertos consolidando los instrumentos finales para su posterior aplicación a los productores de cacao del caserío El Tesoro.

\section{Conclusiones}

Para dar cumplimiento a la última fase del proyecto especial, es 
necesario analizar los datos recolectados por medio de la técnica de la observación y la encuesta de doble entrada, luego, diseñar y aplicar un proceso de la intervención para sistematizar detalladamente cada proceso permitiendo así, cerrar el ciclo investigativo presentando el resultado en un acto público científico sobre las acciones efectuadas con la intención de optimar tanto el método de investigación como la situación observada, generando un producto inédito partiendo de los procesos que se dieron a lo largo del proyecto especial.

Es de hacer notar, que siento este reporte un avance de investigación, no hay resultados concretos hasta la presente, pero se aspira, que se transforme una realidad cultural en cuanto a las técnicas de cultivo del cacao para evitar el deterioro de los suelos en el caserío, además, que el presente trabajo pueda servir como antecedente para futuras investigaciones, que motive a otros investigadores para realizar intervenciones en pro del equilibrio ecológico y finalmente, que pueda servir el caserío El Tesoro como ejemplo a seguir en cuanto a la producción del Cacao por medio de prácticas ecológicas sustentables que protejan los ecosistemas que rodean los cultivos.

\section{Referencias}

Brickell, C.D. et al. (eds) (2009). «International Code of Nomenclature for Cultivated Plants». Scripta Horticulturae. 8th edition. International Society of Horticultural Science, 10: 1-184, ISBN: 978-0-643-09440-6. Spencer y Cross, 2007, p. 938. United States: CSIRO Publishing.

Calva, J. (Coord.), Alba, C., Asuad, N., Bustamante, C., Cardena, F., Carrillo, H... Torres, F. (2007). Sustentabilidad y Desarrollo Ambiental. Agenda para el Desarrollo. Volumen 14, ISBN: 970-32-3532-8. México, D.F.: Dirección General de Publicaciones y Fomento Editorial de la Universidad Nacional Autónoma de México.

Dubois, A. (2007). Producción agrícola y conservación de la 
biodiversidad: ¿Dos actividades compatibles? El caso de los sistemas agroforestales con cacao en Talamanca - Costa Rica. Francia: Université Jean Moulin Lyon 3. Recuperado de:

http://repositorio.bibliotecaorton.catie.ac.cr/handle/11554/3493?show=f ull

Harvey, C., González, J., \& Somarriba, E. (2006). Dung beetle and terrestrial mammal diversity in forests, indigenous agroforestry systems and plantain monocultures in Talamanca, Costa Rica. Biodiversity and Conservation, 15(2), 555-585, e-ISSN: 1572-9710. Recuperado de: https://doi.org/10.1007/s10531-005-2088-2

Hernández, R., Fernández, C., \& Batista, P. (2012). Metodología de la Investigación. $4^{\text {ta }}$ Edición, ISBN: 968-422-931-3. México: McGraw-Hill Interamericana.

ICCO (2006). Informe Anual 2004/2005. Londres, Reino Unido: Organización Internacional del Cacao. Recuperado de:

https://www.icco.org/about-us/international-cocoaagreements/doc download/26-spanish-2004-2005-annual-report.html Cabezas, S., Mavisoy, K., Ballesteros, W., \& Somarriba, E. (2009) Evaluación de la abundancia de Ceratopogonidos (Díptera) polinizadores de cacao (Theobroma cacao L) en la hojarasca de 7 árboles de sombra, Talamanca - Costa rica. Pasto, Colombia: Facultad de Ciencias Agrícolas de la Universidad de Nariño. [Documento en línea]. Recuperado de:

http://biblioteca.udenar.edu.co:8085/atenea/biblioteca/80071.pdf

UPEL (2006a,b,c). Manual de Trabajos de Grado de Especialización y Maestría y Tesis Doctorales. $4^{\text {ta }}$ Edición, ISBN: 980-273-441-1. Caracas, Venezuela: Fondo Editorial de la Universidad Pedagógica Experimental Libertador - FEDUPEL. 


\section{Franklin Rene Guerrero Guerrero \\ e-mail: franklinguerrero77@gmail.com}

Natural del estado Barinas, Venezuela, el 23 de julio del

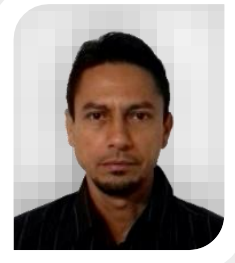
año 1977. Técnico Medio en agropecuaria (Fitotecnia) de la Escuela Técnica Agropecuaria "Pintaderas", ubicada en la Acequia, Municipio Pedraza, Estado Barinas; Ingeniero de Producción Animal de la Universidad Nacional Experimental de los Llanos Occidentales "Ezequiel Zamora" (UNELLEZ, 2009). Entre mis competencias profesionales se pueden destacar mi labor como docente del Ministerio de Educación en Bachillerato en el área agrícola y pecuaria; Tutor de trabajos de Investigación en el área agrícola y pecuaria en la Escuela Técnica Agropecuaria "Don Simón Rodríguez", de igual forma, me desempeño como agricultor de varios rubros, principalmente aguacate, yuca, ñame, plátano y cacao. He realizado conferencias y talleres de formación sobre temas de investigación en cuanto a los cultivos de cacao, por medio de la Universidad Nacional Experimental de los Llanos Occidentales "Ezequiel Zamora" (UNELLEZ), Barinas.

El contenido de este manuscrito se difunde bajo una Licencia de Creative Commons ReconocimientoNoComercial-Compartirlgual 4.0 Internacional 